\title{
Mutation Reporter Tool: An online tool to interrogate loci of interest, with its utility demonstrated using hepatitis B virus
}

Trevor G Bell and Anna Kramvis*

\begin{abstract}
Background: An online tool, which extracts and summarises nucleotide or amino acid sequence data at specified loci of interest, was developed and tested using the basic core promoter/precore (BCP/PC) region of the hepatitis $B$ virus (HBV). The tool is aimed at researchers without specialist computer skills.

Methods: The tool consists of a web-based front-end, with a CGI script, which runs Python code to generate an output web-page. The Python code searches the input sequence data for a specified anchor motif, after which it generates summary tables and graphs of residue and motif distributions.

Results: After the user provides an input file in FASTA format containing aligned sequence data (nucleotides or amino acids) and specifies an anchor motif at a known coordinate, the tool summarizes the nucleotides or amino acids at the specified loci, their frequency and analyzes motif patterns of the loci.The tool can output a graph that displays the frequency of mutations relative to a reference sequence. The tool was used to analyze the BCP/PC region of HBV belonging to subgenotypes A1, A2 and subgenotype D and to serotype HBV. The "Discovery Mode" ignores conserved loci and assists in identifying potential loci of interest.

Conclusions: Although HBV was used to demonstrate the utility of the Mutation Reporter Tool, the tool has wide application as it is genome-agnostic: nucleotide or amino acid sequence data from any organism can be processed. Rapid characterisation of many sequences can be achieved easily when the loci of interest are known. The tool is available online, without charge, at http://hvdr.bioinf.wits.ac.za/tools.
\end{abstract}

Keywords: Hepatitis B virus, Mutations, Sequence analysis

\section{Background}

\section{Example organism: Hepatitis B virus}

Hepatitis B virus (HBV) is one of the most important blood-borne pathogens and is endemic to the sub-Saharan African and southeast Asian regions. Worldwide, around 2 billion people have been exposed to the virus, 240 million are chronically infected, and more than half a million die annually from infection-related liver diseases [1]. At approximately 3,200 nucleotides, the HBV genome is small and has been well-characterized. The genome codes for seven different proteins from four overlapping reading frames (ORFs). To date, nine different genotypes of

\footnotetext{
*Correspondence: Anna.Kramvis@wits.ac.za

Hepatitis B Virus Diversity Research Programme, School of Clinical Medicine,

Faculty of Health Sciences, University of the Witwatersrand, 7 York Road,
}

Parktown, Johannesburg, 2193, South Africa
HBV have been identified: A to D [2,3], E and F [3-5], G [6], H [7], I [8-12] and genotype J has recently been proposed [13]. Subgenotypes have been recogonized in genotypes A to D, F and I, and these are named numerically [14]. Disease progression, clinical manifestation of illness and treatment response differ between these genotypes [15-17].

Mutations (single nucleotide polymorphisms, or "SNPs") in the genetic sequence of HBV are common, as the virus polymerase lacks proof-reading ability [18]. Patterns of mutations at various known loci have been used to characterize the virus [14]. Certain patterns are characterstic of a particular genotype, or subgenotype [14,19], and can therefore be used to identify, or "genotype", a given sample. Patterns at other loci are characteristic of known drug-resistant mutants [20], or indicate other 
important characteristics, such as down-regulation of, for example, hepatitis B e antigen ( $\mathrm{HBeAg})$ [21]. Therefore, the examination of nucleotides at one or more known loci, either together or individually, is routinely used to characterise HBV sequences. Identification of mutations of interest is not always straighforward, however, for a number of reasons. Firstly, the HBV genome is circular (numbered from position "1" at the EcoR1 restriction site), but sequence data is linear, and position " 1 " lies within a region of interest, which is typically sequenced both downstream and upstream of this position. Secondly, HBV genotypes are not the same length, ranging from 3182 , for genotype D, to 3248 nucleotides for genotype G. Thirdly, insertions and/or deletions of varying length may be present in some isolates, or, fourthly, isolates may be recombinants of two or more known genotypes. Thus, automated analysis of the genome is complex and sequence data should be carefully curated.

\section{Basic core promoter/precore (BCP/PC) mutations}

$\mathrm{HBeAg}$ is a non-particulate secretory protein expressed by $\mathrm{HBV}$. The pre-core/core open reading frame encodes for $\mathrm{HBeAg}$ [22]. The basic core promoter (BCP), which covers the distal $\mathrm{X}$ region and the proximal pre-core $(\mathrm{PC})$ region, directs transcription of PC mRNA, which is translated into the pre-core/core fusion protein that is the precursor of HBeAg. This protein has a signal peptide at its amino end that targets it to the endoplasmic reticulum, where it is post-translationally modified by truncation at a fixed site on its amino end and at variable sites on its carboxyl end [21]. Various mutations within the BCP and $\mathrm{PC}$ regions affect the expression of $\mathrm{HBeAg}$ at the transcriptional, translational and post-translational levels $[23,24]$. The BCP A1762T/G1764A mutations affect transcription of the PC mRNA [25]. Mutations that affect $\mathrm{HBeAg}$ expression at the translational level include Kozak sequence (1809-1812) mutations and the G1896A stop codon mutation. Substitutions at 1809-1812 are found mainly in subgenotype A1. HBeAg expression is impaired by Kozak mutations by a leaky scanning mechanism [26]. The classical G1896A transition leads to a tryptophan to stop codon mutation, which results in the truncation of $\mathrm{HBeAg}$ precursor and abrogation of $\mathrm{HBeAg}$ expression [27]. The emergence of G1896A leads to the stabilization of the encapsidation signal $(\varepsilon)$ on the pregenomic RNA in genotypes with $1858 \mathrm{~T}$, but is rarely found in strains which have $1858 \mathrm{C}$ [28]. At the post-translational level, the G1862T mutation, characteteristic of subgenotype A1, introduces a phenylalanine, which interferes with signal peptide cleavage and maturation of $\mathrm{HBeAg}$ [29]. Clinically, $\mathrm{HBeAg}$ is used as an index of viral replication, infectivity, severity of disease and response to antiviral treatment. Mutations that affect HBeAg expression are clinically relevant [17] and thus analysis of their distribution is important. We demonstrate the utility of the Mutation Reporter Tool using the BCP/PC mutations as an example.

\section{Loci of interest and patterns of residues}

Analysis of loci of interest, which may be dispersed across the genome, and the resulting patterns of these loci, has traditionally been a manual, interactive process, which is time-consuming and error-prone. A new online tool, the Mutation Reporter Tool, has been developed to rapidly and easily display loci of interest and patterns of residues for any sequence data (nucleotides or amino acids) submitted by the user. Feedback from members of the Hepatitis Virus Diversity Research Programme, who used development versions of the tool extensively to analyze HBV sequences, was incorporated into the present version.

\section{Results and Discussion}

The Mutation Reporter Tool is one component of a larger project currently in progress, and makes use of a common (shared) Python computer language module, consisting of a "Sequence" class, which contains several methods. The tool consists of a web-based front-end, with which the user interacts, and a CGI script, which runs the Python code and generates the output web-page. The tool has been developed to assist scientists with data analysis and does not require any specialist computer skills or installation. A detailed online tutorial is available. HBV sequence data will be used to demonstrate the utility of the tool.

\section{Usage}

A section of the input interface of the tool is shown in Figure 1. An input file in FASTA format containing aligned sequence data (nucleotides or amino acids) is specified. The loci of interest are specified relative to a known "anchor motif" at a known genomic co-ordinate. The location of the first occurrence of the whole anchor motif in the first sequence in the file is used as the position from which the specified loci are determined. The loci of interest are specified as comma-separated integers without spaces (for example: 1762,1764) and/or dashseparated ranges of integers without spaces (for example: 1809-1812).

For example, the basic core promoter/precore (BCP/ $\mathrm{PC})$ region of $\mathrm{HBV}$ is routinely sequenced. Within this sequence fragment of approximately 500 nucleotides, the highly-conserved motif "AGATTA" is found at coordinate 1750 . A file containing aligned $\mathrm{BCP} / \mathrm{PC}$ sequence data is submitted to the tool with "AGATTA" specified as the anchor motif and "1750" as the anchor position. Loci of interest downsteam of 1750 are then specified by their absolute (and known) co-ordinates in the genome. Loci, which are known to affect the expression of 


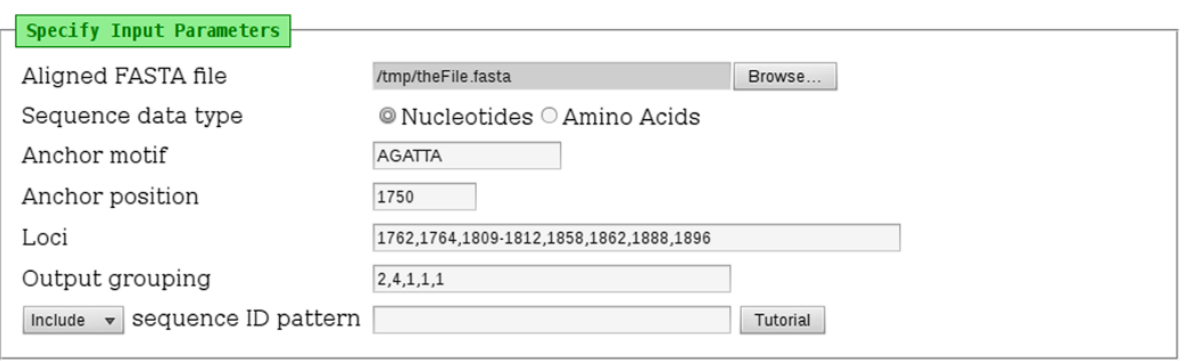

Figure 1 The input interface of the Mutation Reporter Tool. A FASTA input file of sequence data is specified. These sequence data (nucleotides or amino acids) may be genomic or subgenomic fragments. An "anchor motif", which is common to all sequences, is provided. The "anchor position" specifies the genomic co-ordinate of the start of the "anchor motif", such that the downstream loci of interest can be specified accordingly. In this figure, nine loci of interest have been specified. These nine loci will be grouped into columns according to the "output grouping" field: a column containing the first two loci, followed by a column containing the next four loci, followed by a column containing the final locus. Specific sequences in the input file can be included or excluded by entering a regular expression into the appropriate field. This field is blank in the figure, which indicates that all sequences will be included.

$\mathrm{HBeAg}$, are found at 1762, 1764 and 1896. In subgenotype A1, the "Kozak" sequence, which modulates the translation of HBeAg, is located at position "1809-1812". All these loci are therefore entered into the "Loci" field as "1762,1764,1809-1812,1896". Only these loci are extracted from each sequence in the input file and included on the output page.

The loci of interest can optionally be grouped into columns according to the "Output grouping" field. The field accepts a comma-separated list of integers, which indicate the number of loci to group into one output column. If no output grouping is specified, the tool will output all loci into one output column. Using the previous example of loci and an output grouping of " $2,4,1$ ", the output would place the nucleotides at 1762 and 1764 together into one column (specified by the output grouping of " 2 "), the Kozak sequence at 1809-1812 into another column, and nucleotide at 1896 into a third column.

If only some sequences from the input file are to be processed, a "regular expression" can be entered next to the "Include/Exclude" drop-down box. This will then either include (or exclude) sequences for which the FASTA ID matches (or does not match) the regular expression provided. A tutorial describing regular expressions is linked from the input page for reference. Subsets of sequence data stored in one FASTA file can therefore easily be analysed separately, without having to create additional files. FASTA IDs in the output are truncated to the number of characters specified on the input page. If "Output percentages" is not selected, absolute counts are given as output, instead of percentages.

\section{Output}

The tool produces several tables of output. The first (Figure 2) shows the residue at each of the specified loci for each sequence in the input file. The loci are grouped into columns as specified by the output grouping. The next output table shows the distribution of each residue at each locus (as a raw count or a percentage, Figure 3). Figure 4 shows part of the next table of output, which reports the number of occurrences (as a percentage, sorted in ascending order) of each unique motif pattern, as created by placing all of the specified loci next to each other in the order specified. This motif pattern can be used to classify sequences into groups and to identify the motif, which occurs most frequently. A graph of the motif distribution is displayed below the table. The raw data used to create this graph can be downloaded as a CSV file. A link below the final output table (Figure 4) opens a new page, which shows the FASTA ID associated with each of the motif patterns. This output is grouped by motif pattern for reference.

\section{Example Usage: $H B V$ serotypes}

In addition to genotypic classification, HBV strains can be classified into one of nine serological subtypes (serotypes) [30]. This classification is determined by the amino acids

\begin{tabular}{|l|c|c|c|c|c|c|}
\hline ID & 1762 & $\begin{array}{l}1809 \\
1810\end{array}$ & & & & \\
& 1764 & 1811 & 1858 & 1862 & 1888 & 1896 \\
& & 1812 & & & & \\
\hline SHHO01A & AG & TCAT & C & G & G & G \\
\hline SHHO09A & TA & TCAT & C & G & A & G \\
\hline SHHO16A & AG & GCAC & T & G & G & A \\
\hline SHHO22A & AG & TCAT & C & G & A & G \\
\hline SHHO27A & AG & TCAC & T & G & G & A \\
\hline SHHO29A & AG & TCAT & C & G & A & G \\
\hline
\end{tabular}

Figure $\mathbf{2}$ Loci distribution. The nucleotides at each of the specified loci within the BCP/PC region for each sequence in the input file are shown in this table. The loci are grouped into columns as specified. 


\begin{tabular}{|l|l|l|l|l|l|l|l|l|l|l|}
\hline 1762 & 1764 & 1809 & 1810 & 1811 & 1812 & 1858 & 1862 & 1888 & 1896 \\
\hline $\mathbf{A}$ & 083.67 & 018.37 & 004.08 & 002.04 & 085.71 & & & & 059.18 & 010.20 \\
\hline $\mathrm{C}$ & & & & 095.92 & 008.16 & 022.45 & 089.80 & & 002.04 & \\
\hline $\mathbf{G}$ & & 081.63 & 016.33 & & & & & 085.71 & 032.65 & 089.80 \\
\hline $\mathrm{T}$ & 016.33 & & 079.59 & 002.04 & 006.12 & 077.55 & 010.20 & 014.29 & 006.12 & \\
\hline
\end{tabular}

Figure 3 Residue distribution summary. The distribution of residues at each of the loci is shown, either as a percentage or as a raw count, depending on the parameter specified on the input interface (percentages are shown in this figure). Ambiguous (degenerate) bases and gaps, if present in the sequence, would also be included in the table.

present at either three or five known positions within the HBV surface antigen (HBsAg) [31-34]. HBV serotype is loosely correlated with genotype [19]. A published decision tree summarizes the interpretation of the amino acid positions to detemine the HBV serotype [34]. Translated (amino acid) sequence data covering the HBV surface gene can be submitted to the Mutation Reporter Tool with the five amino acid positions of interest (122, 160, 127, 159 and 140) specified. An output grouping of "1,1,1,1,1" should be specified to place each amino acid into its own column for easier reading. The amino acids at each position for each sequence can then be examined together with the decision tree to determine the HBV serotype.

\section{Mutation distribution graph}

A "Reference motif" can be specified on the input page. This motif should include the reference ("wild-type") residue for each of the specified loci, in order. For example, if loci "1809-1812,1896" are specified and the input file consists of HBV subgenotype A1 sequences, the reference motif would be "TCATG". If a reference motif is specified, the output page will include a graph, which indicates the percentage of non-reference (mutant) residues present at each locus. If the input sequence does not contain the ambiguous base " $N$ ", then specifying a reference motif consisting only of " $\mathrm{N}$ " characters will result in the tool including all of the residues at each locus, as all residues will not match the reference residue of "N" at each locus. Additional parameters on the input page are used to customize the graph appearance. These include specifying the graph dimensions (in pixels). Loci at which all sequences contain only the reference residue can be suppressed by selecting the appropriate control on the input page. Selecting the "Y-Axis scaled to 100\%" control will ensure that the Y-axis of the graph extends from $0 \%$ to $100 \%$. This is useful when preparing several graphs which are to be compared with each other. If this control is not selected, the Y-axis will be scaled according to the input data. The raw data used to construct the graph can be downloaded in CSV format from a link on the output page.

\section{Example analysis: Subgenotypes $A 1$ and $A 2$, and genotype $D$}

A comparison of the $\mathrm{BCP} / \mathrm{PC}$ region of subgenotypes $\mathrm{A} 1, \mathrm{~A} 2$ and genotype $\mathrm{D}$ is depicted in Figure 5 . The nucleotide at position 1858 can differentiate between genotypes A and D. Genotype A has $1858 \mathrm{C}$ (Figure 5A and B), whereas genotype D has $1858 \mathrm{~T}$ (Figure 5C). The presence of $1858 \mathrm{C}$ precludes the development of the G1896A mutation because this would destabilize $\varepsilon$ and compromise the replication of the virus [28]. On the other hand, in genotype D, with 1858T, the G1896A mutation would stabilize $\varepsilon$ because of the formation of a Watson-Crick base pair between $1858 \mathrm{~T}$ and 1896A. Thus,

\begin{tabular}{|c|c|}
\hline Label $\longdiv { \text { Motif } }$ & Distribution \\
\hline L0001 AGTCATCGGG & 044.90 \\
\hline 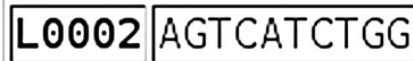 & $01 \odot .20$ \\
\hline L0003 AGGCACCGGG & 006.12 \\
\hline 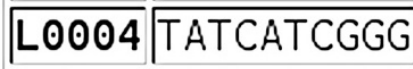 & 006.12 \\
\hline L0005 AGGCACTGGA & $0 \odot 4 . \odot 8$ \\
\hline L0006 TATCTTCGGG & $0 \odot 4.08$ \\
\hline \begin{tabular}{|lll} 
L0007 & AATCCTCGGG \\
\end{tabular} & 002.04 \\
\hline L0008 AGACACCGGG & $0 \odot 2 . \odot 4$ \\
\hline L0009 AGACATCGGG & $0 \odot 2 . \odot 4$ \\
\hline L0010 AGGCACCGGA & 002.04 \\
\hline L0011 AGGCACTTGG & 002.04 \\
\hline L0012 AGTACTTGGA & 002.04 \\
\hline L0013 AGTCACTGGA & 002.04 \\
\hline L0014 AGTCCTCGGG & 002.04 \\
\hline \begin{tabular}{|l|l|l|} 
LOGTCTTCGGG
\end{tabular} & 002.04 \\
\hline \begin{tabular}{|lll} 
L0016 TAGCACCTGG \\
\end{tabular} & 002.04 \\
\hline \begin{tabular}{|l|l|l|l|} 
LATCACCGGG \\
\end{tabular} & 002.04 \\
\hline \begin{tabular}{|l|l|l|} 
TATTCTCGGG \\
\end{tabular} & $0 \odot 2 . \odot 4$ \\
\hline
\end{tabular}

Figure $\mathbf{4}$ Motif distribution. This table shows the distribution, as a percentage, of each unique motif created by placing all the specified loci next to each other in order. 

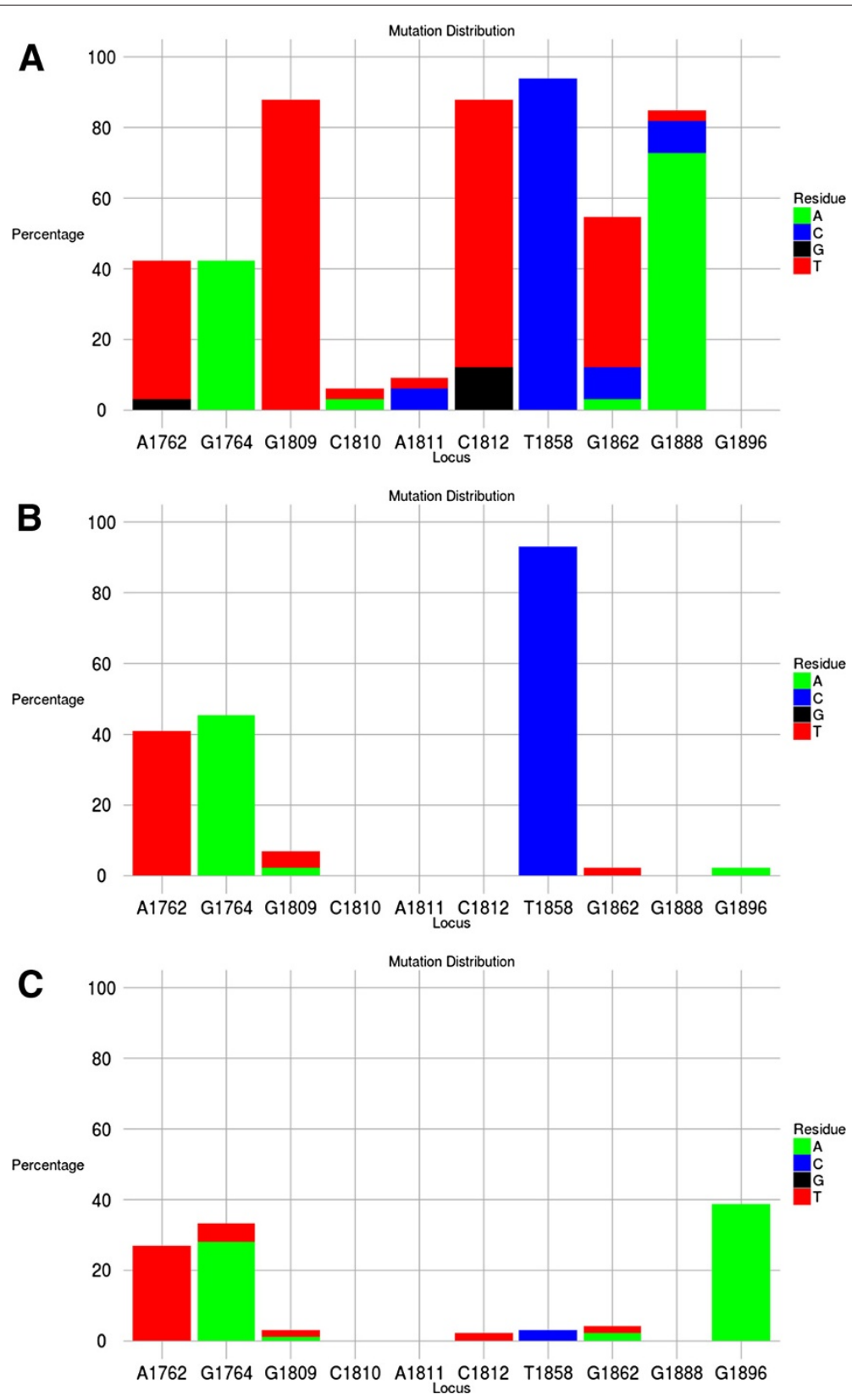

Figure 5 Mutation distribution graph. Mutation distribution graphs showing the percentage of mutant residues relative to the reference motif found at ten loci of interest specified $(1762,1764,1809-1812,1858,1862,1888,1896)$. Three data sets were submitted to the tool to produce the three graphs. Panel A shows the mutation distribution for 33 subgenotype A1 samples, panel B for 34 subgenotype A2 samples and panel C for 93 genotype D samples. The reference motif used was AGGCACTGGG. This is also shown by the letter preceding each locus on the X-axis. To facilitate direct comparisons between the graphs, conserved loci were not suppressd and the $Y$-axis was scaled to $100 \%$ by selecting the appropriate controls on the input page.

as is demonstrated in Figure 5, the G1896A mutation is found in genotype D but not in genotype A. The G1896A leads to a tryptophan to stop codon mutation, which results in the truncation of HBeAg precursor and abrogation of HBeAg expression [27]. G1762T/1764A mutations, which affect the expression of precore mRNA and cause a reduction in $\mathrm{HBeAg}$ expression can develop in genotypes
A and D. However, because G1896A rarely occurs in genotype $\mathrm{A}$, the frequency of $1762 \mathrm{~T} / 1764 \mathrm{~A}$ is higher in this genotype compared to genotype D (Figure 5). This is the only mutation that can affect $\mathrm{HBeAg}$ expression in subgenotype A2 (Figure 5B), whereas in subgenotype $\mathrm{A} 1$ there are additional mutations that can modulate $\mathrm{HBeAg}$ expression (Figure 5A). In subgenotype A1, 
which is also characterized by $1888 \mathrm{~A}$, TCAT instead of GCAC occurs at position 1809-1812 (Figure 5A). This change in the Kozak sequence, preceding the precore start codon at 1814, impairs $\mathrm{HBeAg}$ expression by a leaky scanning mechanism. The effect of the Kozak mutations on $\mathrm{HBeAg}$ expression is comparable with that of the A1762T/G1764A. Co-existence of 1762T1764A and Kozak mutations reduces HBeAg expression in an additive manner [26]. Thus it can be seen that subgenotype A1 has alternative mechanisms for reducing $\mathrm{HBeAg}$ expression compared to genotype $\mathrm{D}$, and subgenotype A2 does not develop mutations that can abrogate HBeAg expression. These differences correlate with the findings of an earlier study, which showed that the prevalence of $\mathrm{HBeAg}$ in serum was significantly lower in carriers of subgenotype A1 than in carriers of A2 or D [35].

\section{Discovery mode}

When the "Discovery Mode" option on the input page is selected, the tool examines the distribution of residues at each of the specified loci and selects for processing only those loci which are not conserved across all input sequences. This mode can be used to "discover" loci of interest by specifying a range of loci, such as "1-100" for example, rather than specific, known loci. The tool will then examine the residues at loci 1 to 100, and will include for further processing and output only those loci at which two or more (different) residues are found. Loci at which only one residue is found will be excluded from the analysis entirely.

When "Discovery Mode" is selected, the "Output grouping", "Reference motif" and graphing parameters are disabled, as the number and position of loci, which would be included in the final analysis is only known after the tool has processed the file. Also, as this is a "discovery mode", it will not be known in advance which loci should logically be grouped together as a unit of interest.

\section{Limitations}

A limitation, by design, is that the sequence data must be aligned. The position of the anchor motif in the first sequence is taken as the anchor position, and loci in all sequences are referenced according to this position. If the input sequence data are not aligned, or if the anchor motif is incorrect, the tool may return incorrect data. Whilst the number of loci, which can be specified, is not limited, it may not be feasible to enter more than a few dozen loci, as this generates a large amount of output data. All loci values must be greater than the anchor position. Updates to the tool will be made to address limitations as necessary.

\section{Conclusions}

As an online tool, available free of charge, no download or installation is required. As demonstrated, this tool can be used for both genotyping and serotyping of HBV without the requirement of computer skills or knowledge of phylogenetics. However, as the tool is genome-agnostic, it has a wide application and nucleotide or amino acid sequence data from any organism can be analysed. Loci of interest, which may be located many hundreds of residues apart, can easily be extracted and their distribution summarised. Rapid characterisation of many sequences, or subsets of sequences, can be achieved easily when the loci of interest are known. Using the "Discovery Mode", conserved and therefore uninformative loci, are automatically ignored, and potential loci of interest can be found and identified.

\section{Methods}

The Mutation Reporter Tool consists of a web-based frontend ("client" interface) with which the user interacts, and a CGI (common gateway interface) script on a server, which runs Python language [36] code to generate the output web-page. The tool is one component of a larger project currently under development, which makes use of a common, shared Python library. The input FASTA file which the user specifies is saved locally (on the server) by the CGI script and then processed by the Python library. Methods within this library are responsible for loading sequence data from a FASTA file, processing the input parameters, and extracting the requested data from the FASTA file. The output HTML page is written to disk by the Python script. The optional output graphs are generated using the ggplot2 graphics library [37] in the $\mathrm{R}$ statistical programming language [38]. If graphs are requested by the user, the Python script writes the relevant data to disk as a CSV (comma-separated value) file. A short R script, which is customized based on the input parameters specified, is also written to disk. The Python script then calls the $\mathrm{R}$ script, which generates the graph and writes it to disk. The images are then linked on the output HTML page. The tool is an online resource, which requires a client browser to connect to the tool's webserver. As such, there is no stand-alone, offline version available for download.

The tool, which assumes that the submitted sequence data is aligned, finds the first occurrence of the anchor motif in the first sequence in the input file. The first character of the anchor motif is then considered to be at the position specified as the anchor position. Sequence data at each of the specified loci for all sequences in the file is then accessed and tabulated. Loci positions are mapped to positions in the sequence data using the anchor motif as an offset value. Data from the loci specified are grouped into columns according to the "output grouping" field. If this field is not specified, all loci are grouped into one output column. If a sequence ID pattern was specified, the tool executes the appropriate regular expression match on 
the FASTA IDs in the input file. In "Discovery Mode", loci at which no variation is found are excluded.

\section{Competing interests}

The authors declare that they have no competing interests.

\section{Authors' contributions}

AK is the principle investigator. TB conceived the idea of the tool, wrote the code, established and maintained the server, software and hardware. TB and AK wrote the paper. Both authors read and approved the final manuscript.

\section{Acknowledgements}

TB received bursaries from the National Research Foundation (NRF), the Medical Research Foundation (MRC), the Poliomyelitis Research Foundation, National Bioinformatics Network and the University of the Witwatersrand. AK received funding from the NRF (GUN\#65530) and the MRC. Mark Keyter and Mukhlid Yousif tested the tool extensively and provided valuable feedback and suggestions.

Received: 16 July 2012 Accepted: 28 January 2013

Published: 23 February 2013

\section{References}

1. WHO: World Health Organization: Hepatitis B fact sheet 204 (July 2012, Revision). 2012. [Accessed on 04 August 2012]. [http://www.who. int/mediacentre/factsheets/fs204/en/]

2. Okamoto H, Tsuda F, Sakugawa H, Sastrosoewignjo Rl, Imai M, Miyakawa $Y$, Mayumi M: Typing hepatitis B virus by homology in nucleotide sequence: comparison of surface antigen subtypes. J Gen Virol 1988 69:2575-2583.

3. Norder H, Couroucé AM, Magnius LO: Complete genomes, phylogenetic relatedness, and structural proteins of six strains of the hepatitis B virus, four of which represent two new genotypes. Virology 1994, 198:489-503.

4. Norder H, Hammas B, Lofdahl S, Courouce AM, Magnius LO: Comparison of the amino acid sequences of nine different serotypes of hepatitis $B$ surface antigen and genomic classification of the corresponding hepatitis B virus strains. J Gen Virol 1992, 73(Pt 5):1201-1208.

5. Naumann H, Schaefer S, Yoshida CF, Gaspar AM, Repp R, Gerlich WH: Identification of a new hepatitis B virus (HBV) genotype from Brazil that expresses HBV surface antigen subtype adw4. J Gen Virol 1993, 74:1627-1632.

6. Stuyver L, Gendt SD, Geyt CV, Zoulim F, Fried M, Schinazi RF, Rossau R: A new genotype of hepatitis $B$ virus: complete genome and phylogenetic relatedness. J Gen Virol 2000, 81:67-74.

7. Arauz-Ruiz P, Norder H, Robertson BH, Magnius LO: Genotype H: a new Amerindian genotype of hepatitis $B$ virus revealed in Central America. J Gen Virol 2002, 83:2059-2073.

8. Olinger CM, Jutavijittum P, Hübschen JM, Yousukh A, Samountry B, Thammavong T, Toriyama K, Muller CP: Possible new hepatitis B virus genotype, southeast Asia. Emerg Infect Dis 2008, 14:1777-1780

9. Tran TTH, Trinh TN, Abe K: New complex recombinant genotype of hepatitis B virus identified in Vietnam. J Virol 2008, 82:5657-5663.

10. Arankalle VA, Gandhe SS, Borkakoty BJ, Walimbe AM, Biswas D, Mahanta J: A novel HBV recombinant (genotype I) similar to Vietnam/Laos in a primitive tribe in eastern India. J Viral Hepatitis 2010, 17:501-510.

11. Osiowy C, Kaita K, Solar K, Mendoza K: Molecular characterization of hepatitis $B$ virus and a 9-year clinical profile in a patient infected with genotype I. J Med Virol 2010, 82:942-948.

12. Yu H, Yuan $\mathrm{O}$ Ge SX, Wang HY, Zhang YL, Chen OR, Zhang J, Chen PJ, Xia NS: Molecular and phylogenetic analyses suggest an additional Hepatitis B virus genotype "I". PLOS One 2010, 5:e9297.

13. Tatematsu K, Tanaka Y, Kurbanov F, Sugauchi F, Mano S, Maeshiro T, Nakayoshi T, Wakuta M, Miyakawa Y, Mizokami M: A genetic variant of hepatitis $B$ virus divergent from known human and ape genotypes isolated from a Japanese patient and provisionally assigned to new genotype J. J Virol 2009, 83:10538-10547.

14. Kramvis A, Arakawa K, Yu MC, Nogueira R, Stram DO, Kew MC Relationship of serological subtype, basic core promoter and precore mutations to genotypes/subgenotypes of hepatitis B virus. J Med Virol 2008, 80:27-46.
15. Mayerat C, Mantegani A, Frei PC: Does hepatitis B virus (HBV) genotype influence the clinical outcome of HBV infection? J Viral Hepatitis 1999, 6:299-304

16. Sumi H, Yokosuka O, Seki N, Arai M, Imazeki F, Kurihara T, Kanda T, Fukai K Kato M, Saisho H: Influence of hepatitis B virus genotypes on the progression of chronic type B liver disease. Hepatology 2003 , 37:19-26.

17. Kramvis A, Kew MC: Relationship of genotypes of hepatitis B virus to mutations, disease progression and response to antiviral therapy. J Viral Hepatitis 2005, 12:456-464.

18. Steinhauer DA, Holland JJ: Direct method for quantitation of extreme polymerase error frequencies at selected single base sites in viral RNA. J Virol 1986, 57:219-228.

19. Kramvis A, Kew M, François $G$ : Hepatitis B virus genotypes. Vaccine 2005, 23:2409-2423.

20. Zoulim F, Locarnini S: Hepatitis B virus resistance to Nucleos(t)ide analogues. Gastroenterology 2009, 137:1593-1608.

21. Revill $P$, Yuen $L$, Walsh R, Perrault M, Locarnini S, Kramvis A: Bioinformatic analysis of the hepadnavirus e-antigen and its precursor identifies remarkable sequence conservation in all orthohepadnaviruses. J Med Virol 2010, 82:104-115.

22. Ou JH, Laub O, Rutter WJ: Hepatitis B virus gene function: the precore region targets the core antigen to cellular membranes and causes the secretion of the e antigen. Proc Nat Acad Sci USA 1986, 83:1578-1582.

23. Kramvis A, Kew MC: Epidemiology of hepatitis B virus in Africa, its genotypes and clinical associations of genotypes. Hepatology Res 2007, 37:S9-S19.

24. Tong $S$ : Impact of viral genotypes and naturally occurring mutations on biological properties of hepatitis B virus. Hepatology Res 2007, 37:S3-S8.

25. Buckwold VE, Xu Z, Chen M, Yen TS, Ou JH: Effects of a naturally occurring mutation in the hepatitis $B$ virus basal core promoter on precore gene expression and viral replication. J Virol 1996, 70:5845-5851.

26. Ahn SH, Kramvis A, Kawai S, Spangenberg HC, Li J, Kimbi G, Kew M, Wands $J$, Tong S: Sequence variation upstream of precore translation initiation codon reduces hepatitis $B$ virus e antigen production. Gastroenterology 2003, 125:1370-1378.

27. Carman WF, Hadziyannis S, Mcgarvey MJ, Jacyna MR, Karayiannis P, Makris A, Thomas HC: Mutation preventing formation of hepatitis B e antigen in patients with chronic hepatitis B infection. Lancet 1989 334:588-591.

28. Lok AS, Akarca U, Greene S: Mutations in the pre-core region of hepatitis B virus serve to enhance the stability of the secondary structure of the pre-genome encapsidation signal. Proc Nat Acad Sci USA 1994, 91:4077-4081.

29. Chen CY, Crowther C, Kew MC, Kramvis A: A valine to phenylalanine mutation in the precore region of hepatitis $B$ virus causes intracellular retention and impaired secretion of $\mathrm{HBe}$-antigen. Hepatology Res 2008, 38:580-592.

30. Magnius LO, Norder H: Genotypes and molecular epidemiology of the Hepatitis B virus as reflected by sequence variability of the S-Gene. Intervirology 1995, 38:24-34

31. Wands JR, Wong MA, Shorey J, Brown RD, Marciniak RA, Isselbacher KJ: Hepatitis B viral antigenic structure: signature analysis by monoclonal radioimmunoassays. Proc Nat Acad Sci USA 1984 81:2237-2241.

32. Mimms LT, Floreani M, Tyner J, Whitters E, Rosenlof R, Wray L, Goetze A, Sarin $\mathrm{V}$, Eble K: Discrimination of hepatitis B virus (HBV) subtypes using monoclonal antibodies to the PreS1 and PreS2 domains of the viral envelope. Virology 1990, 176:604-619.

33. Swenson PD, Riess JT, Krueger LE: Determination of HBsAg subtypes in different high risk populations using monoclonal antibodies. J Virological Methods 1991, 33:27-38.

34. Purdy MA, Talekar G, Swenson P, Araujo A, Fields A: A new algorithm for deduction of Hepatitis $B$ surface antigen subtype determinants from the amino acid sequence. Intervirology 2007, 50:45-51.

35. Tanaka Y, Hasegawa I, Kato T, Orito E, Hirashima N, Acharya SK, Gish RG, Kramvis A, Kew MC, Yoshihara N, Shrestha SM, Khan M, Miyakawa Y, Mizokami M: A case-control study for differences among hepatitis B 
virus infections of genotypes $A$ (subtypes $A a$ and $A e$ ) and $D$.

Hepatology 2004, 40:747-755.

36. van Rossum G: Python. [http://www.python.org]

37. Wickham H: ggplot2: Elegant Graphics for Data Analysis. New York: Springer; 2009.

38. R Core Team: R: A Language and Environment for Statistical Computing. Vienna: R Foundation for Statistical Computing; 2012. [http://www.Rproject.org/].

doi:10.1186/1743-422X-10-62

Cite this article as: Bell and Kramvis: Mutation Reporter Tool: An online tool to interrogate loci of interest, with its utility demonstrated using hepatitis B virus. Virology Journal 2013 10:62.

Submit your next manuscript to BioMed Central and take full advantage of:

- Convenient online submission

- Thorough peer review

- No space constraints or color figure charges

- Immediate publication on acceptance

- Inclusion in PubMed, CAS, Scopus and Google Scholar

- Research which is freely available for redistribution

Submit your manuscript at www.biomedcentral.com/submit
( BioMed Central 